\title{
To Tweet or Not to Tweet-a Review of the Viral Power of Twitter for Infectious Diseases
}

\author{
Ravina Kullar ${ }^{1}$ - Debra A. Goff ${ }^{2} \cdot$ Timothy P. Gauthier $^{3} \cdot$ Tara C. Smith $^{4}$ \\ Published online: 24 April 2020 \\ (C) Springer Science+Business Media, LLC, part of Springer Nature 2020
}

\begin{abstract}
Purpose of Review With real-time communication crucial to both healthcare professionals (HCPs) and the public in infectious diseases (ID), social media networking sites has become even more important. Twitter is the most popular form of social media used for ID communication. We will review the power of Twitter in ID.

Recent Findings Twitter allows for real-time sharing of educational resources at ID scientific conferences, enabling individuals that are not able to attend conferences to follow conferences anytime anywhere and stimulate discussion around topics of interest with experts from across the globe. Further, Twitter chats are a valuable tool for stewardship, with different accounts periodically hosting chats on various stewardship topics. Several studies have also demonstrated the strong relationship between dissemination and citation impact of publications with the help of Twitter. There is great value in engaging with non-ID people on Twitter via dissemination of ID knowledge to other disciplines. Lastly, when used appropriately, Twitter is a useful site for distributing vaccine information, whether informally (by advocates and physicians) or formally (by government entities) and allows one to keep up with ongoing ID outbreaks in real time.

Summary Twitter has transformed how we communicate in healthcare. Particularly in ID, where bacteria and viruses can enter/ exit borders anytime anywhere, global real-time information about outbreaks and antimicrobial resistance for clinicians and the public is critical. Twitter has no hierarchy or barriers, is a conduit for global collaboration, and is a way for HCPs and the public to "social"ize on healthcare topics, if used appropriately.
\end{abstract}

Keywords Twitter · Antimicrobial stewardship $\cdot$ Vaccines $\cdot$ Infectious diseases $\cdot$ Social media $\cdot$ Coronavirus $\cdot$ COVID19

This article is part of the Topical Collection on Technology and Infectious Disease

Ravina Kullar

ravina.kullar@gmail.com

Debra A. Goff

Debbie.Goff@osumc.edu

Timothy P. Gauthier

timothypgauthier@gmail.com

Tara C. Smith

tsmit176@kent.edu

1 Expert Stewardship, Inc, 320 Superior Ave, Newport Beach, CA 92663, USA

2 The Ohio State University College of Pharmacy, The Ohio State University Wexner Medical Center, Columbus, OH, USA

3 Baptist Health South Florida, Miami, FL, USA

4 Kent State University College of Public Health, Kent, OH, USA

\section{Introduction}

Social media networking sites are playing a prominent role in healthcare - these sites are web-based services where users create a public or semi-public profile, generate a list of users, and then view a list of connections and posts formed by others within a system [1]. Based on a report published by the Pew Research Center, $87 \%$ of the total USA population used the Internet, with the offline population gradually declining from $48 \%$ of the total population in 2000 to $13 \%$ of the total population in 2016. [2] According to a telephone survey conducted in 2010 by the Pew Internet Project and California Health Care Foundation, searching for health or medical information is one of the most popular activities online, with $80 \%$ of Internet users, or about 93 million Americans, having searched for a health-related topic online [3].

New medical information is typically acquired via reading scientific journals and attending conferences. However, realtime communication is critical to both healthcare professionals 
(HCPs) and the public to distribute medical alerts and information. Currently, Twitter is the most popular form of social media used for healthcare communication [4]. Twitter (founded in July 2006) is a microblogging version of a social media site where users interact in real time via posts ("tweets") containing up to 280 characters; hashtags (\#) within these posts allow aggregation of tweets into topics. Users interact with each other through updates, direct messaging, replies, likes, and retweeting, which is the reposting of specific messages. There is no permission needed to follow a user, and reciprocation of following is not required. In its first-quarter statistics for 2019, Twitter declared 330 million active monthly users [5]. Twitter has become a daily part of many HCPs' lives, with 152 million people daily users, allowing them to communicate real-time healthcare information and medical alerts to a large global audience, including those who are considered experts or thought leaders in a particular field, and to solicit feedback [5].

Social media is particularly paramount in infectious diseases as real-time reporting to alert and empower relevant public health surveillance systems, authorities, and the public about taking the optimal and necessary actions to control and minimize the potential harmful effects of infectious diseases is critical. The role of Twitter in infectious diseases has been previously studied, with Twitter being useful in monitoring the spread of the swine flu (H1N1) outbreak, which was declared a pandemic in 2009, as well as studying and identifying the misuse of antibiotics [6]. During the 2014 Ebola outbreak in West Africa, from July 24, 2014-August 1, 2014 (9 days), 42,236 tweets mentioning Ebola were posted and disseminated to $9,362,267,048$ people in real time, spreading breaking health news, with most of these tweets sent 3 days before the official outbreak announcements [7]. With the USA being at the brink of losing its measles elimination status in 2019, Twitter served as a powerful platform to disseminate correct information about vaccines [8]. Most recently, with the coronavirus (COVID19) outbreak in 2020, Twitter enabled the public and HCPs to stay up-to-date on the case and death numbers worldwide, as the world inched closer to another pandemic. Twitter has also been crucial for engaging, educating, and advocating for the global public health crisis of antimicrobial resistance (AMR) [9, 10]. Accordingly, in this review, we will highlight the value of Twitter in infectious diseases, providing research studies and relevant examples from Twitter.

\section{Conferences}

Twitter has been used for several years to extend the reach of scientific conferences, with growing success. At the 2014 annual Making a Difference in Infectious Diseases conference, which is focused on providing antimicrobial stewardship (AMS) educational topics for physicians and pharmacists, a workshop on Twitter for Infectious Diseases Healthcare
Providers was held. The conference planners registered the hashtag \#MADID14 with the Healthcare Hashtag Project [11]. Individuals at the conference signed up for Twitter and then learned how to follow each other and tweet live from the conference. A total of 235 participants sent 1482 tweets during the 3-day conference. Attendees were able to connect with thought leaders and, more importantly, sustain the connection through Twitter after the meeting. Further, tweets incorporating the hashtag \#asm2012, representing the meeting of the American Society of Microbiology, became so popular that it was listed as a "trending" topic on the Twitter home page [12]. When the Oral versus Intravenous Antibiotics for Bone and Joint Infection (OVIVA) study was first presented at the 2017 European Congress of Clinical Microbiology \& Infectious Disease (ECCMID) [13], clinicians from around the world, whether they were at the conference or not, were able to see the presented slides years before the study was even published and without traveling to Europe. Since publication of the trial [14], the authors have engaged with individuals on Twitter to answer questions about the results of the study. In effect, Twitter allows for quick real-time sharing of educational resources at conferences, enabling individuals that are not able to attend the conference to follow the conference from a distance at any time and stimulating discussion around topics of interest with experts from across the globe. With the help of Twitter, learning is no longer restricted by the time or geographical limitations of the conference.

\section{Twitter Chats and Dissemination of Infectious Diseases Literature}

Twitter chats have emerged as a valuable tool for AMS, and there are several accounts that periodically host Twitter chats on AMS topics. During a Twitter chat, a central account Tweets questions or topics with a common theme (e.g., penicillin allergies, outpatient antimicrobial stewardship) and then individuals interested in the topic can contribute via tweeting their perspectives, advice, and helpful resources or publications. These chats are coordinated through the use of a central hashtag that allows people to follow the conversation. Twitter chats can be daylong events or more frequently last for $1 \mathrm{~h}$ with a new Tweet released from the central account approximately every $10 \mathrm{~min}$.

One recurring Twitter chat focused on AMS is the \#ASPchat hosted by @ASP_chat. The \#ASPchat was inspired by World Antibiotic Awareness Week, began in December 2016, and occurs on the third Thursday of every month. It is co-hosted by an AMS pharmacist in Canada (@BRxAD) and the USA (@IDstewardship), periodically collaborating with professional organizations with shared interests (e.g., Society for Healthcare Epidemiology of America (SHEA), National Collaborating Centre for Infectious Diseases (NCCID)). To date, there have been 35 \#ASPchat sessions with an average 
of 329 tweets and 81 participants per chat. Participants are frequently medical professionals (e.g., physicians, pharmacists) located in North America; however, there have been participants from many countries outside of North America and even The Joint Commission has participated. Cumulatively, the \#ASPchat has achieved over 23,000,000 impressions, with an upward trend per chat over time (Fig. 1). These impressions have been calculated through use of the Symplur Healthcare Hashtags Project data source. Symplur calculates total impressions by taking the number of tweets per participant and multiplying it with the number of followers that participant currently has.

Twitter can serve as a pivotal platform for disseminating new publications and, sometimes, articles will even circulate on Twitter prior to the authors being aware the publication is available e-pub online. Staying current with literature is a challenge, but when the new publications shared on Twitter populate directly into your feed and some of the authors are available to interact with on Twitter, it is all much easier to follow and more engaging. Accordingly, a recent study explored the role of scientific journals on Twitter, analyzing how their articles were tweeted and cited. Over 4000 research articles from 350 scientific journals were extracted, with results revealing that articles from journals with their own account were $46 \%$ more tweeted and $34 \%$ more cited than articles from journals without a Twitter account. Similar findings were found in a study evaluating the impact of authors having Twitter accounts in dissemination of articles, revealing that scientific papers from Twitter users were $33 \%$ more tweeted those of non-Twitter users [15]. These studies demonstrate the strong relationship between dissemination and citation impact with the help of Twitter [16].

Relevant to this topic, an AMS pharmacist from Saudi Arabia (@ABsteward) and the USA (@IDstewardship) have teamed up to summarize important new AMS and infectious diseases publications, releasing an article with links to all of the top latest publications of the previous month. The authors of this series have seen significant growth in their followers in the past year and release of each article garners significant attention on social media. The 17 articles published to date have averaged 2683 pageviews per article with over 43,000 cumulative pageviews. Not all pageviews are the direct result of Twitter users; nevertheless, this exemplifies yet another avenue where Twitter can be valuable for AMS.

\section{Value of Twitter Beyond Infectious Disease Specialists}

Many ID physicians and pharmacists join Twitter to follow other ID colleagues or "like" people. While there is great value in engaging with people who have similar interests, there is equal value in engaging with "unlike" or non-ID people. For example, how do surgeons keep current on emerging drugresistant pathogens and learn about new antibiotics? Most surgeons are not reading the latest ID journals for this information. Surgeons need timely education on multidrugresistant organisms to prescribe effective antibiotics. Twitter can provide a vehicle for surgeons to learn about emerging new drug-resistant pathogens in real time.

One study evaluated Twitter as a tool to engage and educate surgeons in emerging ID topics on AMR and AMS. ID pharmacists tweeted ID topics relevant to surgeons, and each tweet's reach, impressions, and engagement were calculated [17]. Surgeons engaged in 72 ID tweets for a reach of 5101, impressions of 38,784 , and actual impressions of 15,895 . The most re-tweeted tweet was on the University of California Los Angeles outbreak of carbapenem-resistant Klebsiella pneumonia transmitted by contaminated duodenoscopes during endoscopic retrograde cholangiopancreatography
Fig. 1 \#ASPchat impressions over time
\#ASPchat Impressions Over Time

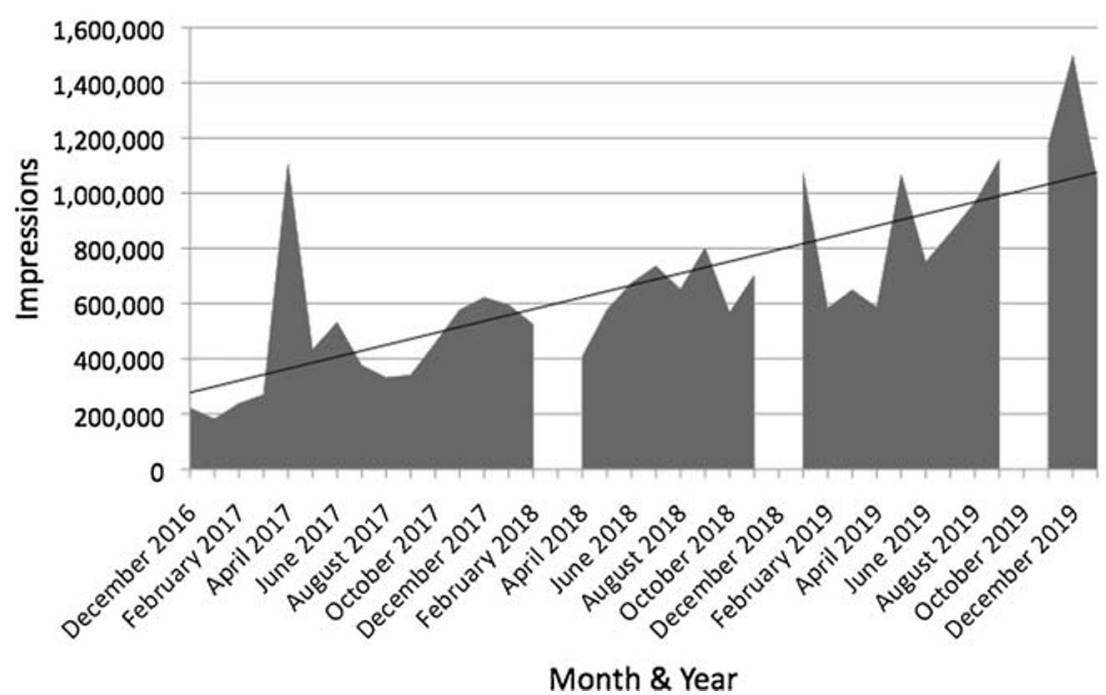


procedures. Over 3000 people viewed the tweet, which was linked to the Centers for Disease Control and Prevention (CDC) guidance document on how to manage these patients.

The study proved ASP pharmacists and physicians could use Twitter to educate surgeons in real time. Targeted tweets with ID topics of interest to surgeons can continue to build engagement with surgeons globally. One ID pharmacist (@idpharmd) uses this approach to engage and educate surgeons on ID topics. As a result of the connection via Twitter, she was invited to speak on a global surgical webinar with over 450 surgeons from around the world [18].

\section{Patient Advocacy}

Twitter is frequently used by patients or families that have been impacted by AMR or antibiotic-associated adverse events. For example, the Peggy Lillis Foundation uses Twitter to educate consumers on the risk of acquiring $C$. difficile infection from antibiotics. Vanessa Carter, who survived a methicillin-resistant Staphylococcus aureus (MRSA) infection and became an ePatient Scholar, tweets regularly to over 5400 followers. She is also the founder of \#hcsmSA which is an acronym for Health Care Communications and Social Media South Africa. Carter was invited as a civil guest to present her e-Patient story for a meeting at the Africa CDC/African Union in Ethiopia which focused on the implementation of the Antimicrobial Resistance Strategy for Africa and is currently an e-Patient Scholar at Stanford University Medicine. Further, Mary Millard contracted a Pseudomonas aeruginosa (P. aeruginosa) infection that caused her to go into septic shock. She was featured on the Infectious Diseases Society of America (IDSA) Patient Stories and uses Twitter to engage people in the sepsis conservation and to increase people's knowledge of how AMR can gravely impact one's life.

\section{Vaccines and Outbreak Information}

In social media, vaccines have become a hot topic, as is evident from a tweet posted by a microbiologist with only a few thousand followers receiving over 75,000 retweets and over 300,000 likes in just a 3-day period. Reaching this level of attention is almost unheard of for most of the ID-related content on Twitter [19]. In 2019, for the first time in history, the World Health Organization (WHO) ranked vaccine hesitancy, a "reluctance or refusal to vaccinate despite the availability of vaccines," among the top ten global health threats, along with Ebola, $\mathrm{HIV}$, and drug-resistant infections [20]. Twitter can serve as a useful site for dissemination of vaccine information, whether informally (by advocates and physicians) or formally (by government entities). One example of vaccine advocacy that has had remarkable influence is Jill Promoli's work, via her personal account (@jillpromoli) and her organization's site (@4jude4everyone, \#ForJudeForEveryone). Promoli lost her son, Jude, to an influenza B infection in 2015 [21] and has since become an advocate for the influenza vaccination. In 2018 and 2019, Canadian Prime Minister Justin Trudeau shared his influenza vaccination picture on Twitter, tagging Promoli and @ 4Jude4everyone in his picture [22, 23]. Chelsea Clinton (@ChelseaClinton) similarly shared the \#forjudeforeveryone hashtag in 2017, urging everyone to receive a flu shot [24].

Twitter can also serve as a conduit to keep up with an ongoing outbreak situation. In Samoa, a small Pacific island country of about 200,000 people, a measles outbreak exploded in late 2019, after a traveler from New Zealand entered the country in mid-August. The outbreak went undetected until mid-October, and then quickly spread through the population, which had a very low vaccination rate. By November 11 , there had been 628 cases and 5 deaths; by January 6 of 2020, 5697 measles cases had been reported, with 83 deaths [25]. Twitter acted as both a repository of information and as an avenue to discuss the outbreak, for both the public and clinicians. The Government of Samoa (@samoagovt) tweeted out details of the outbreak almost daily beginning on November 22, adding additional information on their Facebook page (https://www. facebook.com/samoagovt). This allowed scientists and others on Twitter to discuss the outbreak developments in real time, to craft epidemic curves [26], and to develop timelines of the outbreak in order to dispel the misinformation that the outbreak was caused by the vaccine [27].

At this time in early 2020, the SARS-CoV-2 (termed COVID19) epidemic is increasing, but Twitter has also been a go-to source of information on the virus and the outbreak. The public and HCPs get timely updates on the epidemic through Twitter, with the WHO even live streaming their daily updates via Twitter. Many users have curated lists of reputable accounts to follow who are experts in the field of infectious disease, virology, epidemiology, clinical medicine, health policy, and more, such as this list by epidemiologist Ellie Murphy (@EpiEllie, https://twitter.com/i/lists/1220869298631200769). The World Health Organization (@WHO) and Centers for Disease Control and Prevention (@CDCgov) have been actively tweeting breaking information about the outbreak. Additionally, if you enter "coronavirus" into the Facebook search function, the first result directs users in most countries globally to go to the WHO for the latest information.

Moreover, Dr. Thomas File (@theIDFile),whowas appointed the President of IDSA in October 2019, put out the following message about vaccines: "One of the significant accomplishments of the year is the successful Why I Vaccinate twitterstorm IDSA created during IDWeek 2019. Being a Twitter novice myself, trust me when I tell you how amazed I was at the level of activity around an issue that is so important to all of us. Vaccine hesitancy threatens to undo so many of the greatest accomplishments of our field. As ID professionals, we have an obligation to turn the tide on this frightening trend, and I think we made a major statement within a 
platform that is too often used to spread misinformation. By all of us coming together to share our own personal messages about why we believe in vaccination, we were the \#1 trending hashtag in Washington, DC, \#5 in the nation, and \#28 worldwide. We will continue to make our voices heard on this critical issue in the months and years ahead including pursing legislation for vaccine access and education."

\section{Limitations}

Since no systematic process exists to check for incorrect material on Twitter, social media can be a landmine of misinformation. For instance, with vaccines, while Facebook [28], Instagram [29], and Pinterest [30] have made advances to reduce the anti-vaccine information available on their platforms; overall, there has still not been substantial progress in reducing anti-vaccine content on these platforms [31]. Similarly, Twitter is the major social media platform for leading anti-vaccine organizations in the USA and remains a go-to site for convening anti-vaccine political activism and for promoting misinformation. Twitter's only pushback against such misinformation has been to redirect individuals searching for vaccine-associated keywords to a reputable site (vaccines.gov, run by the US Department of Health and Human Services) [32]. Unfortunately, the anti-vaccine movement has gained traction and doubt in vaccines has grown [33], in part because of misguided tweets and the anti-vaccine posts not being policed by the site and not reportable as a group.

Exacerbating the COVID19 outbreak has been misinformation, which has spread quicker through social media channels, such as Twitter, than COVID19 on the ground - being termed an "infodemic." This has led to confusion and panic by the public. For instance, politicians and even scientists spread unintentional misinformation and fear (https://www.theatlantic.com/ technology/archive/2020/01/china-coronavirus-twitter/605644/). It is important for individuals to go to credible resources such as @WHO, @CDCgov, and @IDSAInfo.

Further, due to the nature of Twitter, responding to such misinformation can be a difficult choice. As a public site, any reader can see responses to posts. Even when misinformation is corrected, the mere exposure to such material may cause it to spread [34]. As such, countering misinformation should be performed with consideration. Additionally, those tweeting pro-vaccine information are vulnerable to attacks by antivaccine activists and/or bots. A pediatrician was recently the center of attacks after posting a TikTok video, a video-sharing app, encouraging vaccination on Twitter. It took less than $24 \mathrm{~h}$ for the video to go viral on both TikTok and Twitter - and just another $48 \mathrm{~h}$ before the physician was facing backlash and death threats from people associated with the anti-vaccine movement [35]. Recently, an account was developed in order to "rally the troops" in support of an individual under attack by anti-vaccine accounts: @shotsheard. [36]

\section{Conclusions}

The use of social media platforms such as Twitter has transformed the manner in which we communicate on both a personal and professional level. The way we stay up-to-date has mirrored these advancements. Particularly in infectious diseases, where bacteria and viruses can enter and exit borders anytime anywhere, global real-time information about outbreaks and AMR for both clinicians and the public is critical. Twitter has no hierarchy or barriers, serves as a conduit for global collaboration, and is a way for both HCPs and the public to "social"ize on healthcare topics, if used appropriately.

Acknowledgments We acknowledge Dr. Brad Langford (@BRxAD) for his role in the \#ASPchat and Dr. Bassam Ghanem (@ABsteward) for his role in the monthly antimicrobial stewardship literature summaries. We also acknowledge Dr. Peter Hotez for the review of our paper.

\section{Compliance with Ethical Standards}

Conflict of Interest Ravina Kullar, Debra A. Goff, Timothy P. Gauthier, and Tara C. Smith declare that they have no conflicts of interest.

Human and Animal Rights and Informed Consent This article does not contain any studies with human or animal subjects performed by any of the authors.

Disclaimer The views and opinions expressed in the article and on social media are those of the authors and do not necessarily reflect the position or policy of their past, present, or potential future employers. This article was completed outside of working hours without the use of company resources.

\section{References}

1. Boyd DM, Ellison NB. Social network sites: definition, history and scholarship. J Comput-Mediat Commun. 2007;13:210-30.

2. Anderson M, Perrin A. Pew research. $13 \%$ of Americans don't use the Internet. Who are they? 2016 Available at: http://www. pewresearch.org/fact-tank/2016/09/07/some-americans-dont-usethe-internet-who-are-they/. Accessed December 1, 2019.

3. Pew Research Center: Internet and Technology. Health Topics. Available at: https://www.pewresearch.org/internet/2011/02/01/ health-topics-2/ Accessed December 1, 2019.

4. Raghupathi W, Raghupathi V. Big data analytics in healthcare: promise and potential. Health Inf Sci Syst. 2014;2:3. https://doi. org/10.1186/2047-2501-2-3.

5. Business of Apps. Twitter Revenue and Usage Statistics. 2019. Available at: https://www.businessofapps.com/data/twitterstatistics/ Accessed December 1, 2019.

6. Kostova P, de Quincey E, Jawaheer G. The potential of social networks for early warning and outbreak detection systems: the swine flu Twitter study. Int J Infect Dis. 2010;14:e384-5.

7. Odlum M, Yoon S. What can we learn about the Ebola outbreak from tweets? Am J Infect Control. 2015;43(6):563-71. https://doi. org/10.1016/j.ajic.2015.02.023. 
8. Centers for Disease Control and Prevention. Measles. Available at: https://www.cdc.gov/measles/elimination.html. Accessed February 25, 2020.

9. Goff DA, Kullar R, Laxminarayan R, Mendelson M, Nathwani D, Osterholm M. Twitter to engage, educate, and advocate for global antibiotic stewardship and antimicrobial resistance. Lancet Infect Dis. 2019;19(3):229-31. https://doi.org/10.1016/S1473-3099(19) 30058-1.

10. Goff DA, Kullar R, Newland JG. Review of Twitter for infectious diseases clinicians: useful or a waste of time? Clin Infect Dis. 2015;60(10):1533-40. https://doi.org/10.1093/cid/civ071.

11. Symplur. Available at: http://www.symplur.com/healthcarehashtags/madid14/. Accessed December 5, 2019.

12. Bik HM, Goldstein MC. An introduction to social media for scientists. PLoS Biol. 2013;11(4):e1001535. https://doi.org/10.1371/ journal.pbio.1001535.

13. Scarborough M, Li HK, Rombach I, et al. The OVIVA Trial: oral versus intravenous antibiotics in the management of bone and joint infection - a multicentre open label randomised non-inferiority study. [Abstract \#OSO42]. 27th ECCMID; Vienna, Austria: 2225 April 2018.

14. Li HK, Rombach I, Zambellas R, Walker AS, McNally MA, Atkins $\mathrm{BL}$, et al. Oral versus intravenous antibiotics for bone and joint infection. N Engl J Med. 2019;380(5):425-36. https://doi.org/10. 1056/NEJMoa1710926.

15. Ortega JL Scientometrics. 2016;109:1353. https://doi.org/10.1007/ s11192-016-2113-0.

16. Ortega J. The presence of academic journals on Twitter and its relationship with dissemination (tweets) and research impact (citations). Aslib J Inf Manag. 2017;69(6):674-87. https://doi.org/10. 1108/AJIM-02-2017-0055.

17. Goff DA, Jones C, Toney B, et al. Use of Twitter to educate and engage surgeons in infectious diseases and antimicrobial stewardship. Infect Dis Clin Pract. 2016;24(6):324-7. https://doi.org/10. 1097/IPC.0000000000000440.

18. The Global Alliance for Infections in Surgery. Available at: https:// infectionsinsurgery.org/free-webinar-meeting/. Acccessed February 26, 2020

19. Twitter. Available at: https://twitter.com/richdavisphd/status/ 1217493336342163456?s=20. Accessed on January 17, 2020.

20. World Health Organization. Ten Threats to Global Health in 2019. Available at: https://www.who.int/emergencies/ten-threats-toglobal-health-in-2019 Accessed January 5, 2020.

21. Dunham J. CTVNews. Toronto-area mother launches flu vaccine campaign after toddler's death. Available at: https://www.ctvnews. $\mathrm{ca} /$ health/toronto-area-mother-launches-flu-vaccine-campaignafter-toddler-s-death-1.3130727. Accessed January 8, 2020.
22. Twitter. Available at: https://witter.com/justintrudeau/status/ 1065609458410246144. Accessed January 8, 2020.

23. Twitter. Available at: https://twitter.com/JustinTrudeau/status/ 1196953162822930434. Accessed January 8, 2020.

24. Twitter. Available at: https://twitter.com/chelseaclinton/status/ 930214653678047233. Accessed January 8, 2020.

25. Twitter. Available at: https://witter.com/samoagovt/status/ 1214429581760819200. Accessed January 8, 2020.

26. Twitter. Available at: https://witter.com/maiamajumder/status/ 1203079477967966209. Accessed January 8, 2020.

27. Twitter. Available at: https://twitter.com/MackayIM/status/ 1211494983095570433. Accessed January 8, 2020.

28. Bickert M. Combatting vaccine misinformation. Facebook newsroom. 2019. Available at: https://newsroom.fb.com/news/2019/03/ combatting-vaccine-misinformation/. Accessed January 8, 2020.

29. Naftulin J. Instagram banned anti-vaccine messaging, but antivaxxers are hijacking pro-choice hashtags to game the system. Available at: https://www.insider.com/anti-vaxxers-are-findinginstagram-loopholes-to-spread-dangerous-message-2019-12. Accessed January 8, 2020.

30. Pinterest. Available at: https://policy.pinterest.com/en/communityguidelines. Accessed January 8, 2020.

31. BuzzFeed.News. Facebook is running anti-vax ads, despite its ban on vaccine misinformation. Available at: https://www. buzzfeednews.com/article/carolinehaskins 1/facebook-runninganti-vax-ads-despite-ban-anti. Accessed January 10, 2020.

32. Harvey D. Helping you find reliable public health information on Twitter. Available at: https://blog.twitter.com/en us/topics/ company/2019/helping-you-find-reliable-public-healthinformation-on-twitter.html. Accessed January 10, 2020.

33. Reinhart RJ. Gallup: Fewer in U.S. Continue to See Vaccines as Important. 2020. Available at: https://news.gallup.com/poll/ 276929/fewer-continue-vaccines-important.aspx. Accessed January 13, 2020.

34. Pluviano S, Watt C, Della SS. Misinformation lingers in memory: failure of three pro-vaccination strategies. PLoS One. 2017;12(7): e0181640. https://doi.org/10.1371/journal.pone.0181640.

35. Twitter. Available at: https://twitter.com/NicoleB_MD/status/ 1216124793801662464. Accessed January 15, 2020.

36. Twitter. Available at: https://twitter.com/shotsheard. Accessed January 8, 2020.

Publisher's Note Springer Nature remains neutral with regard to jurisdictional claims in published maps and institutional affiliations. 